\title{
The perioperative management of anticoagulants and antiplatelet agents in cervical and adrenal surgery
}

\author{
Niki Christou ${ }^{1,2 *}$ and Sébastien Gaujoux ${ }^{3,4}$ \\ ${ }^{1}$ Department of Endocrine General and Digestive Surgery, Universitary Hospital of Limoges, 2 rue Martin Luther King, 87042 Limoges cedex, France \\ ${ }^{2}$ Faculty of Medicine and Pharmacy, University of Limoges, 2 rue du Dr Marcland, 87025 Limoges cedex, France \\ ${ }^{3}$ Department of Hepato-biliary and Endocrine Digestive Surgery, AP-HP, Cochin Hospital, 27 rue du Faubourg Saint Jacques, 75014 Paris Paris, France \\ ${ }^{4}$ University Paris Descartes, Paris, France
}

\begin{abstract}
Nowadays, there is no review of the literature on current recommendations concerning the perioperative management of anticoagulants and antiplatelet agents in endocrine surgery - particulary concerning cervical and adrenal surgery. Moreover, this is true since the introduction of new oral anticoagulants.

As a consequence, the purpose of the present review tries to make an update of recommendations concerning the perioperative management of anticoagulants and antiplatelet agents in the specific context of endocrine surgery.
\end{abstract}

\section{Introduction}

Cervical surgery, thyroid or parathyroid one, has a low morbidity. One of the main risks is post-operative hematoma, which, when compressive, can lead to a very short-term life expectancy in the event of delayed diagnosis or management. In this context, the perioperative management of anticoagulants and antiplatelet agents is particularly important.

In adrenal surgery, if the occurrence of a postoperative hematoma is not usually associated with an immediate life-threatening risk, this risk may in particular be influenced by the secreting nature of the lesions, which will necessitate an adaptation of the antithrombotic prophylaxis.

\section{Thromboembolic prophylaxis}

\section{Cervical surgery}

All the studies revealed a significantly lower thromboembolic risk than hemorrhagic risk, even after surgery for cancer. There is therefore no indication of systematic post-operative medicinal thromboembolic prophylactic treatment [1]. This can be individually indicated according to particular thromboembolic and haemorrhagic risk factors [2,3]. (Level of evidence: 4). In major carcinological surgery, which may involve, for example, tracheal or esophageal resection, prophylaxis is permissible because of the duration of surgery and especially of the field $[1,4]$.

\section{Adrenal Surgery}

By laparotomy: No specific recommendations were made for thrombo-prophylaxis in adrenal surgery. Thus, to give the indication, the search for known individual thromboembolic risk factors such as obesity, thromboembolic history, cancer, female sex, advanced age, prolonged duration of intervention, varicose veins, can help us [5]. According to the recommendations of SFAR 2005, and by analogy with open or endo-urological surgery of the upper urinary tract, adrenal surgery is considered to be a low thrombotic surgical risk and does not require specific thrombo-prophylaxis. Whether in the absence or presence of individual thromboembolic risk factors, anti-thrombosis stockings (ATS) are recommended [1] (grade A). It is important to note that patients with Cushing's syndrome or who are operated from cancer have a higher risk of thromboembolism and therefore require systematic post-operative thromboembolic prevention for a duration of 2 weeks [6] (level of evidence: 2).

By laparoscopy: Despite its classification in the major hemorrhagic surgery group [7], most adrenal surgeries are performed by laparoscopy with minimal blood loss [8]. The recommendations are identical to laparotomy except in case of laparoscopy more than 60 minutes where the thrombotic surgical risk becomes moderate. In this last case, a pair of ATS or heparin therapy with LMWH (Low Molecular Weight Heparinotherapy) (grade D), except in case of cancer, where prolongation of prophylaxis at one month reduced paraclinic thrombosis by $50 \%$ without an increase in haemorrhagic risk (Level of evidence 1). In the presence of individual thromboembolic risk factors, LMWHs are recommended (grade D).

\section{Perioperative management in long-term patients}

- by antiplatelet agents

\section{Cervical surgery}

There is no indication of discontinuation of acetylsalicylic acid therapy that does not significantly increase hemorrhagic and

${ }^{\star}$ Correspondence to: Christou Niki, Endocrine General and Digestive Surgery Department, Universitary Hospital of Limoges, 2 rue Martin Luther King, 87042 Limoges cedex, France, E-mail: christou.niki19@gmail.com

Key words: anticoagulants; antiplatelet agents; endocrine surgery

Received: August 06, 2018; Accepted: August 15, 2018; Published: August 20 2018 
postoperative hematoma risks, nor does transfusion risk (Level of evidence: 4).

P2Y12 receptor blockers (clopidogrel, prasugrel, ticagrelor) should be discontinued and relieved by aspirin, knowing that they are rarely given alone but most often with aspirin for patients with a coronary stent [9] (Level of evidence: 4). In the event of stoppage, the total duration of the treatment should not exceed 3 days for clopidogrel and ticagrelor, 5 days for acetylsalicylic acid and 7 days for prasugrel. In all cases, a multidisciplinary decision with the cardiologist or neurologist must be obtained. For example, aspirin is stopped 2 days before surgery and taken again 24 hours later.

In addition, a reinforced surveillance must be carried out within the 24 hours following the gesture in search of an evocative sign of postoperative hematoma [9] (Level of evidence: 4).

Systematic drainage of the surgical site does not reduce the risk of postoperative hematoma [9] (Level of evidence: 4).

\section{Adrenal Surgery}

According to HAS - ANSM 2012 guidelines [9], hemorrhagic risk in adrenal surgery is low. The discontinuation of antiplatelet agents such as acetylsalicylic acid is therefore not justified. Clopidogrel, prasugrel and ticagrelor should be stopped at a greater risk of bleeding, but should be relayed by acetylsalicylic acid. Their resumption should be considered quickly in light of the benefit-risk balance. The monitoring of the thrombotic risk, particularly coronary perioperative risk, must be established in collaboration with the anesthetic team and the physicians initiating the treatment.

- by anticoagulants

\section{Cervical surgery}

It is recommended, when possible $[10,11]$, to stop effective anticoagulant therapy before cervicotomy. (Grade 1C). The Antivitamin $\mathrm{K}$ (AVK) should be stopped 5 days before the procedure, the Dabigatran, 48 hours before.

\section{Adrenal Surgery}

It is recommended, where possible $[10,11]$ to stop effective anticoagulant therapy before adrenalectomy. (Grade 1C).

If it is possible to stop therapeutic anticoagulation by AVK without relay [11].

- The last AVK dose should be given 5 days before surgery.

- Perform INR dosage the day before the intervention for goal of INR $<1.5$ with vitamin $\mathrm{K}$ prescription, oral if necessary, with a verification of 6 hours.

- LMWH anticoagulation should be resumed cautiously within 12 to $24 \mathrm{~h}$ after surgery (next morning) if hemostatic control is correct, gradually increasing doses to achieve therapeutic anticoagulation between 48 th and 72 th hour.

- If it's necessary to stop therapeutic anticoagulation by AVK and perform relay by heparin [11]:

- Relay with therapeutic dose of Unfractionated heparin (UFH) should be stopped 4 to 6 hours before surgery.

- Relay with therapeutic dose of LMWH should be stopped 24 hours before surgery.
- LMWH or UFH should not be resumed at a fixed time after surgery or procedure without an examination of the risk of premature bleeding or the relevance of postoperative haemostasis.

- If the therapeutic dose relay is used in patients at high risk of postoperative bleeding, initiation should be delayed 48 to 72 hours after surgery when adequate surgical hemostasis has been achieved. If the bleeding persists beyond $72 \mathrm{~h}$, the options include a low-dose heparin relay or AVK alone with no post-operative heparin relay. In other cases, the therapeutic dose heparin relay can be done 24 hours after surgery.

\section{Outpatient surgery}

Cervical surgery- The last recommendations of the French Association of Endocrine Surgery (AFCE) [12] are not in favor, without totally contraindicating it, of ambulatory surgery after cervicotomy. The need for antiplatelet therapy or effective anticoagulant therapy is clearly contraindications to ambulatory care, and is in agreement with the recommendations of the American Thyroid Association (ATA) [13] (Level of evidence: 1 ).

Adrenal Surgery- Nowadays, there is very little experience with ambulatory laparoscopic adrenalectomy, and there is no recommendation of learned society. In this context, the need for antiplatelet therapy or effective anticoagulant therapy seems contraindications to ambulatory care. (Level of evidence: 4).

\section{Acknowledgements}

We thank for the proofreading the anesthesists, Professors Samama (University Hospital Cochin, Paris) and Nathan-Denizot (University Hospital Dupuytren, Limoges).

\section{Disclosure statement}

No competing financial interests exist.

\section{References}

1. SFAR (2005) Prevention Recommendations for Perioperative and Obstetrical Venous Thromboembolic Disease Available at: http://sfar.org/wp-content/uploads/2015/09/2 AFAR_Prevention-de-la-maladie-thromboembolique-veineuse-perioperatoire-etobstetricale.pdf [Accessed 14 July 2017]

2. Roy M, Rajamanickam V, Chen H, Sippel R (2010) Is DVT prophylaxis necessary for thyroidectomy and parathyroidectomy? Surgery 148: 1163-1168. [Crossref]

3. Verma KP, Gorelik A, Miller JA (2013) Thyroid Surgery and Anticoagulation: Survey of Practice of Endocrine Surgeons in Australia. World Journal of Endocrine Surgery. 1: 6-11.

4. SFAR (2011) Prevention Recommendations for Perioperative and Obstetrical Venous Thromboembolic Disease Available at: http://sfar.org/wp-content/uploads/2015/10/2 AFAR_Prevention-de-la-maladie-thromboembolique-veineuse-postoperatoire-copie.pdf

5. Nomura T, Takahashi M, Iwasaki K (2013) Efficacy and Safety of Enoxaparin for Preventing Venous Thromboembolic Events following Urologic Laparoscopic Surgery. Int Sch Res Not 2: e415918. [Crossref]

6. Manetti L, Bogazzi F, Giovannetti C (2010) Changes in coagulation indexes and occurrence of venous thromboembolism in patients with Cushing's syndrome: results from a prospective study before and after surgery. Eur J Endocrinol 163: 783-91.

7. Heidbuchel H, Verhamme P, Alings M (2015) Updated European Heart Rhythm Association Practical Guide on the use of non-vitamin K antagonist anticoagulants in patients with non-valvular atrial fibrillation. Europace 17: 1467-1507. [Crossref]

8. Shah DR, Wang H, Bold RJ, Yang X, Martinez SR, et al. (2013) Nomograms to predict risk of in-hospital and post-discharge venous thromboembolism after abdominal and thoracic surgery: an American College of Surgeons National Surgical Quality Improvement Program analysis. J Surg Res 183: 462-471. [Crossref]

9. SFAR (2012) Recommandations de bonne pratique- bon usage des agents antiplaquettaires - ANSM-HAS- Juin. 
10. Godier A, Pernod G, Sié P (2008) Perioperative management of avk: recommendations. MAPAR 29: 331-339

11. Douketis JD, Spyropoulos AC, Spencer FA (2012) Perioperative management of antithrombotic therapy: Antithrombotic Therapy and Prevention of Thrombosis, 9th ed: American College of Chest Physicians Evidence-Based Clinical Practice Guidelines. Chest 141: 326-350. [Crossref]
12. Menegaux F (2013) Outpatient thyroidectomy: recommendations from the Francophone Association of Endocrine Surgery (AFCE). Survey of current practices. $J$ Chir Viscérale 150:185-192.

13. Terris DJ, Snyder S, Carneiro-Pla D (2013) American Thyroid Association Statement on Outpatient Thyroidectomy. Thyroid 23: 1193-1202. [Crossref]

Copyright: $\odot 2018$ Christou N. This is an open-access article distributed under the terms of the Creative Commons Attribution License, which permits unrestricted use, distribution, and reproduction in any medium, provided the original author and source are credited. 